\title{
One-Dimensional Time-Dependent Frustrated and Disordered Ising Model
}

\author{
M. B£AsZYK \\ Institute of Physics, A. Mickiewicz University \\ Umultowska 85, 61-614 Poznań, Poland \\ (Received March 20, 2007; revised version March 3, 2008; \\ in final form May 12, 2008)
}

\begin{abstract}
The dynamics being modifications of the Glauber one were proposed. The time course of the single spin and two-spin correlation function was determined. These calculations were performed for ordered systems, although a possibility of their extension over disordered ones was indicated.
\end{abstract}

PACS numbers: 05.50.+q, 75.10.Nr, 75.50.Lk

\section{Introduction}

We study the combined effects of disorder and frustration on time dependences of thermodynamical quantities. We consider the kinetic Ising model in the version proposed by Glauber [1]. This model has already been considered at different levels of complexity. Admission of a possibility of the exchange integrals taking random values complicates the problem, although it does not introduce competition, but it can still be analytically investigated ([2] and [3]). Additional complexity appears when frustration is admitted. The simplest model with frustration is the one-dimensional antiferromagnetic Ising model in a magnetic field. However, in this model only for zero magnetic field it is possible to derive expressions for the time dependences of thermodynamic quantities, as only then the time derivative of the $n$-spin correlation function can be expressed in terms of the corresponding $n$-spin correlation functions. For other models the equation of motion of the $n$-spin correlation function includes also other correlations, which means that all $2^{N}$ correlation functions must be taken into regard to derive an expression for anyone of them. In literature this rule is known as the BBGKY hierarchy [4].

In certain particular cases the disordered one-dimensional Ising model has been analysed in an external magnetic field. The case when the magnetic field $H$ can be assumed to tend to zero has been studied by José et al. [5]. A radically different situation of the random fields of infinite values when the system 
undergoes fragmentation has been considered by Forgacs et al. [6]. Analysis of the dynamics of the one-dimensional Ising model in a random magnetic field has been made within the mean field approximation by Luque and Cordoba [7]. As the one-dimensional ordered Ising model in a magnetic field is the simplest case whose exact description requires consideration of all $2^{N}$ equations of motion, attempts have been made at its investigation by Hilhorst [8], Baumgärtner and Binder [9]. The price that has been paid for this analysis was the necessity to describe the kinetics by the clusters of spins of the same values and to abandon the simple language of the mean correlation functions used by Glauber, which led to a numerical analysis. Therefore, computer simulations still remain the most effective tool in investigation of the dynamics of disordered systems with competitive interactions. For example, Das and Barma [10] studied the Ising model to which frustration is introduced by addition of the second nearest neighbours to the chain.

In this paper a weakening of the detailed balance condition has been proposed, as a result of which the systems that could not have been solved by the Glauber approach have become available for symbolic analysis (1D Ising model in a magnetic field, 1D Ising model with the first and second neighbours). The approach in which one spin is left out in the master equation is called the dynamics with a one-spin window, while the approach with two spins left out is called the dynamics with a two-spin window. The possibility of applying this formalism for description of diluted models has been given.

\section{Dynamics with a single-spin window}

Analogously as in [1] and [11], our considerations will begin with the master equation for the probability of the spins taking the values $s_{1}, \ldots, s_{N}$ at time $t$ :

$$
\begin{aligned}
& \frac{\mathrm{d}}{\mathrm{d} t} P\left(s_{1}, \ldots, s_{N}, t\right)=-\sum_{j} W\left(s_{j}\right) P\left(s_{1}, \ldots, s_{j}, \ldots, s_{N}, t\right) \\
& \quad+\sum_{j} W\left(-s_{j}\right) P\left(s_{1}, \ldots,-s_{j}, \ldots, s_{N}, t\right) .
\end{aligned}
$$

Multiplying the equation by $s_{k}$ and summing over the spin states we get

$$
\frac{\mathrm{d}}{\mathrm{d} t}\left\langle s_{k}\right\rangle=-2\left\langle s_{k} W\left(s_{k}\right)\right\rangle \text {. }
$$

It is assumed for $W\left(s_{k}\right)$ that in the equilibrium the condition of the detailed balance is fulfilled

$$
W\left(s_{j}\right) P\left(s_{1}, \ldots, s_{j}, \ldots, s_{N}, t\right)=W\left(-s_{j}\right) P\left(s_{1}, \ldots,-s_{j}, \ldots, s_{N}, t\right) .
$$

We weaken this condition making the summation over all spins except the $j$-th one

$$
W\left(s_{j}\right) P\left(s_{j}, t\right)=W\left(-s_{j}\right) P\left(-s_{j}, t\right) .
$$

Although in Eqs. (3) and (4) the same notation $W\left(s_{j}\right)$ was used for the probability of the $j$-th spin changing the sign from $s_{j}$ to $-s_{j}$ in a time unit, the form of the expressions is different. For the case of only the nearest neighbours interactions, 
in Eq. (4) the probability $W\left(s_{j}\right)$ is a function of only one spin $s_{j}$, while in Eq. (3) it depends on the spin $s_{j}$ and also on its neighbours $s_{j-1}$ and $s_{j+1}$. The above weakening of condition (3), whose consequences will be discussed later, leads to the equilibrium state, although the dynamics is changed. Let us consider a 1D Ising chain of $N$ sites in an external magnetic field $H$. It is described by the Hamiltonian

$$
\mathcal{H}=-\sum_{i} J_{i, i+1} s_{i} s_{i+1}-H \sum_{i} s_{i}
$$

where $s_{i}= \pm 1$. The element of the transition matrix $\left\langle s_{i}\right| \exp \left(\beta J_{i, i+1} s_{i} s_{i+1}+\right.$ $\left.\frac{1}{2} \beta H\left(s_{i}+s_{i+1}\right)\right)\left|s_{i+1}\right\rangle$ is proportional to the probability of a pair of spins taking the values $s_{i}$ and $s_{i+1}$. In order to determine this probability we find the $n$-th power of the transition matrix. Let us denote by $\boldsymbol{M}$ the transition matrix for one bond

$$
\boldsymbol{M}=\left[\begin{array}{ll}
\mathrm{e}^{K+B} & \mathrm{e}^{-K} \\
\mathrm{e}^{-K} & \mathrm{e}^{K-B}
\end{array}\right]
$$

where $K=\beta J, B=\beta H$ and $\beta=1 / k T$. Then following the similarity transformation

$$
\boldsymbol{A}^{-1} \boldsymbol{M A}=\left[\begin{array}{ll}
\lambda_{+} & 0 \\
0 & \lambda_{-}
\end{array}\right]
$$

where $\lambda_{ \pm}=\mathrm{e}^{K} \operatorname{ch} B \pm \sqrt{\mathrm{e}^{2 K} \operatorname{sh}^{2} B+\mathrm{e}^{-2 k}}$, permits expressing $\boldsymbol{M}^{N}$ in the form

$$
\boldsymbol{M}^{N}=\frac{1}{y}\left[\begin{array}{cc}
\lambda_{+}^{N} \mathrm{e}^{2 K}(\operatorname{sh} B+x)-\lambda_{-}^{N} \mathrm{e}^{2 K}(\operatorname{sh} B-x) & \lambda_{+}^{N}-\lambda_{-}^{N} \\
\lambda_{+}^{N}-\lambda_{-}^{N} & \lambda_{+}^{N} \mathrm{e}^{2 K}(-\operatorname{sh} B+x)-\lambda_{-}^{N} \mathrm{e}^{2 K}(\operatorname{sh} B+x)
\end{array}\right],
$$

where $y=2 \mathrm{e}^{2 K} x$ and $x=\sqrt{\operatorname{sh}^{2} B+\mathrm{e}^{-4 K}}$. The probability that spins 1 and $N+1$ are upwards is directly proportional to $\left\langle+\left|\boldsymbol{M}^{N}\right|+\right\rangle$, while the probability that these spins are downwards is proportional to $\left\langle-\left|\boldsymbol{M}^{N}\right|-\right\rangle$. Having imposed the periodic conditions on the chain considered, putting $s_{1}=s_{N+1}$ and the probability normalisation condition, the probabilities of the $s_{1}$ spin directed upwards $P\left(s_{1}=1\right)$ or downwards $P\left(s_{1}=-1\right)$ can be written as

$$
\begin{aligned}
& P\left(s_{1}=1\right)=\frac{\left\langle+\left|M^{N}\right|+\right\rangle}{\left\langle+\left|M^{N}\right|+\right\rangle+\left\langle-\left|M^{N}\right|-\right\rangle}, \\
& P\left(s_{1}=-1\right)=\frac{\left\langle-\left|M^{N}\right|-\right\rangle}{\left\langle+\left|M^{N}\right|+\right\rangle+\left\langle-\left|M^{N}\right|-\right\rangle} .
\end{aligned}
$$

With these probabilities we can get the following expression from Eq. (4):

$$
W\left(s_{j}\right)=\frac{1}{2}-\frac{1}{2} s_{j} \frac{\operatorname{sh} B}{\sqrt{\operatorname{sh}^{2} B+\mathrm{e}^{-4 K}}} \frac{\lambda_{+}^{N}-\lambda_{-}^{N}}{\lambda_{+}^{N}+\lambda_{-}^{N}} .
$$

This choice of the transition probability leads to the following equation for the mean spin: 


$$
\frac{\mathrm{d}}{\mathrm{d} t}\left\langle s_{i}\right\rangle=-\left\langle s_{i}\right\rangle+\frac{\operatorname{sh} B}{\sqrt{\operatorname{sh}^{2} B+\mathrm{e}^{-4 K}}} \frac{\lambda_{+}^{N}-\lambda_{-}^{N}}{\lambda_{+}^{N}+\lambda_{-}^{N}} .
$$

By putting $K=0$ then $\lambda_{+}=2 \operatorname{ch} B, \lambda_{-}=0$ and Eq. (11) can be rewritten as

$$
\frac{\mathrm{d}}{\mathrm{d} t}\left\langle s_{i}\right\rangle=-\left\langle s_{i}\right\rangle+\tanh B
$$

In this form it is identical with Eq. (79) derived by Glauber [1]. For $B=0$ and a finite $K$ we get $\frac{\mathrm{d}}{\mathrm{d} t}\left\langle s_{i}\right\rangle=-\left\langle s_{i}\right\rangle$, but after taking the limits in the sequence $N \rightarrow \infty$, $K \rightarrow \infty$ and $B \rightarrow 0$, then $\frac{\mathrm{d}}{\mathrm{d} t}\left\langle s_{i}\right\rangle=-\left\langle s_{i}\right\rangle+1$. Therefore, our model always tends to the state $\left\langle s_{i}\right\rangle=0$ except for the case when $N \rightarrow \infty, K \rightarrow \infty$ and $B \rightarrow 0$ it tends to $\left\langle s_{i}\right\rangle=1$. For the Glauber model $\frac{\mathrm{d}}{\mathrm{d} t}\left\langle s_{i}\right\rangle=-\left\langle s_{i}\right\rangle+\frac{1}{2} \tanh 2 K\left(\left\langle s_{i-1}\right\rangle+\left\langle s_{i+1}\right\rangle\right)$, so in order to compare the two models we impose the translational invariance of the system $\left\langle s_{i-1}\right\rangle=\left\langle s_{i}\right\rangle=\left\langle s_{i+1}\right\rangle$ then $\frac{\mathrm{d}}{\mathrm{d} t}\left\langle s_{i}\right\rangle=-\left\langle s_{i}\right\rangle(\tanh 2 K-1)$. Irrespective of the number of sites, the system tends to the state with $\left\langle s_{i}\right\rangle=0$, for finite $K$ and to the state of indefinite $\left\langle s_{i}\right\rangle$ when $K \rightarrow \infty$. Thus, we can conclude that although the equations of motion or other dynamics are different, in both cases the system tends to the same equilibrium state. The solution of Eq. (11) can be written as

$$
\left\langle s_{i}\right\rangle(t)=\left\langle s_{i}\right\rangle\left(t_{0}\right) \mathrm{e}^{-\left(t-t_{0}\right)}+\frac{\operatorname{sh} B}{\sqrt{\operatorname{sh}^{2} B+\mathrm{e}^{-4 K}}} \frac{\lambda_{+}^{N}-\lambda_{-}^{N}}{\lambda_{+}^{N}+\lambda_{-}^{N}}\left(1-\mathrm{e}^{-\left(t-t_{0}\right)}\right) .
$$

In order to find the correlation $\left\langle s_{j} s_{k}\right\rangle$ the detailed balance condition (3) is multiplied by $s_{k}$ and a summation is made over all spin states besides $s_{j}$, which gives

$$
s_{k} W\left(s_{j} ; s_{k}\right) P\left(s_{j}, s_{k}\right)=s_{k} W\left(-s_{j} ; s_{k}\right) P\left(-s_{j}, s_{k}\right) .
$$

Calculation of $\boldsymbol{X}=\boldsymbol{M}^{N}$ and $\boldsymbol{Y}=\boldsymbol{M}^{k} \boldsymbol{S} \boldsymbol{M}^{N-k}$, where $\boldsymbol{S}$ is the spin matrix, permits writing $s_{k} P\left(s_{0}, s_{k}\right)$ in the form

$$
\begin{aligned}
s_{k} P & \left(s_{0} ; s_{k}\right)=\frac{1}{2}\left(1+s_{0}\right) \frac{\langle+|\boldsymbol{Y}|+\rangle}{\langle+|\boldsymbol{X}|+\rangle+\langle-|\boldsymbol{X}|-\rangle} \\
& +\frac{1}{2}\left(1-s_{0}\right) \frac{\langle-|\boldsymbol{Y}|-\rangle}{\langle+|\boldsymbol{X}|+\rangle+\langle-|\boldsymbol{X}|-\rangle} \\
& =\frac{1}{2} \frac{\langle+|\boldsymbol{Y}|+\rangle+\langle-|\boldsymbol{Y}|-\rangle}{\langle+|\boldsymbol{X}|+\rangle+\langle-|\boldsymbol{X}|-\rangle}+\frac{1}{2} s_{0} \frac{\langle+|\boldsymbol{Y}|+\rangle-\langle-|\boldsymbol{Y}|-\rangle}{\langle+|\boldsymbol{X}|+\rangle+\langle-|\boldsymbol{X}|-\rangle},
\end{aligned}
$$

where

$$
\begin{aligned}
& \langle+|\boldsymbol{Y}|+\rangle+\langle-|\boldsymbol{Y}|-\rangle=\frac{\operatorname{sh} B}{\sqrt{\operatorname{sh}^{2} B+\mathrm{e}^{-4 K}}}\left(\lambda_{+}^{N}-\lambda_{-}^{N}\right), \\
& \langle+|\boldsymbol{Y}|+\rangle-\langle-|\boldsymbol{Y}|-\rangle=\frac{1}{\mathrm{e}^{4 K}\left(\mathrm{sh}^{2} B+\mathrm{e}^{-4 K}\right)} \\
& \quad \times\left[\lambda_{+}^{k} \lambda_{-}^{N-k}+\lambda_{-}^{k} \lambda_{+}^{N-k}+\mathrm{e}^{4 K} \operatorname{sh}^{2} B\left(\lambda_{+}^{N}-\lambda_{-}^{N}\right)\right], \\
& \langle+|\boldsymbol{X}|+\rangle+\langle-|\boldsymbol{X}|-\rangle=\lambda_{+}^{N}+\lambda_{-}^{N} .
\end{aligned}
$$

As follows from the above definitions:

$$
\sum_{s_{0}= \pm 1} s_{k} P\left(s_{0} ; s_{k}\right)=\left\langle s_{k}\right\rangle=\frac{\langle+|\boldsymbol{Y}|+\rangle+\langle-|\boldsymbol{Y}|-\rangle}{\langle+|\boldsymbol{X}|+\rangle+\langle-|\boldsymbol{X}|-\rangle}
$$




$$
=\frac{\operatorname{sh} B}{\sqrt{\operatorname{sh}^{2} B+\mathrm{e}^{-4 K}}} \frac{\lambda_{+}^{N}-\lambda_{-}^{N}}{\lambda_{+}^{N}+\lambda_{-}^{N}}
$$

and the transition probability can be written as

$$
\begin{gathered}
W\left(s_{j} ; s_{k}\right)=W\left(s_{j} ; s_{-k}\right)=\frac{1}{2} p-\frac{1}{2} s_{j} q=\frac{1}{2} \frac{\operatorname{sh} B}{\sqrt{\operatorname{sh}^{2} B+\mathrm{e}^{-4 K}}} \frac{\lambda_{+}^{N}-\lambda_{-}^{N}}{\lambda_{+}^{N}+\lambda_{-}^{N}} \\
-\frac{1}{2} s_{j} \frac{\lambda_{+}^{k-j} \lambda_{-}^{N-k+j}+\lambda_{-}^{k-j} \lambda_{+}^{N-k+j}+\mathrm{e}^{4 K} \operatorname{sh}^{2} B\left(\lambda_{+}^{N}+\lambda_{-}^{N}\right)}{\mathrm{e}^{4 K}\left(\operatorname{sh}^{2} B+\mathrm{e}^{-4 K}\right)\left(\lambda_{+}^{N}+\lambda_{-}^{N}\right)} .
\end{gathered}
$$

From this expression the equation of motion for $\left\langle s_{j} s_{k}\right\rangle$ is obtained

$$
\begin{aligned}
& \frac{\mathrm{d}}{\mathrm{d} t}\left\langle s_{j} s_{k}\right\rangle(t)=\frac{\mathrm{d}}{\mathrm{d} t}\left[\sum_{s_{j}= \pm 1} s_{j} s_{k} P\left(s_{j}, t ; s_{k}\right)+\sum_{s_{k}= \pm 1} s_{j} s_{k} P\left(s_{k}, t ; s_{j}\right)\right] \\
& =\sum_{s_{j}= \pm 1} s_{j} s_{k}\left[-W\left(s_{j} ; s_{k}\right) P\left(s_{j}, t ; s_{k}\right)+W\left(-s_{j} ; s_{k}\right) P\left(-s_{j}, t ; s_{k}\right)\right] \\
& +\sum_{s_{k}= \pm 1} s_{j} s_{k}\left[-W\left(s_{k} ; s_{j}\right) P\left(s_{k}, t ; s_{j}\right)+W\left(-s_{k} ; s_{j}\right) P\left(-s_{k}, t ; s_{j}\right)\right] \\
& =\sum_{s_{j}= \pm 1} s_{j} s_{k}\left[\frac{1}{2}\left(-p+s_{j} q\right) P\left(s_{j}, t ; s_{k}\right)+\frac{1}{2}\left(p+s_{j} q\right) P\left(-s_{j}, t ; s_{k}\right)\right] \\
& +\sum_{s_{k}= \pm 1} s_{j} s_{k}\left[\frac{1}{2}\left(-p+s_{k} q\right) P\left(s_{k}, t ; s_{j}\right)+\frac{1}{2}\left(p+s_{k} q\right) P\left(-s_{k}, t ; s_{j}\right)\right] \\
& =-2 p\left\langle s_{j} s_{k}\right\rangle(t)+q\left[\left\langle s_{j}\right\rangle(t)+\left\langle s_{k}\right\rangle(t)\right] .
\end{aligned}
$$

As we have already determined $\left\langle s_{i}\right\rangle(t)$ (13), Eq. (18) can be written as

$$
\begin{aligned}
& \frac{\mathrm{d}}{\mathrm{d} t}\left\langle s_{j} s_{k}\right\rangle(t)=-\frac{2 \operatorname{sh} B}{\sqrt{\operatorname{sh}^{2} B+\mathrm{e}^{-4 K}}} \frac{\lambda_{+}^{N}-\lambda_{-}^{N}}{\lambda_{+}^{N}+\lambda_{-}^{N}}\left\langle s_{j} s_{k}\right\rangle(t) \\
& +\frac{\lambda_{+}^{k-j} \lambda_{-}^{N-k+j}+\lambda_{-}^{k-j} \lambda_{+}^{N-k+j}+\mathrm{e}^{4 K} \operatorname{sh}^{2} B\left(\lambda_{+}^{N}+\lambda_{-}^{N}\right)}{\mathrm{e}^{4 K}\left(\operatorname{sh}^{2} B+\mathrm{e}^{-4 K}\right)\left(\lambda_{+}^{N}+\lambda_{-}^{N}\right)} \\
& \times\left\{\left[\left\langle s_{j}\right\rangle\left(t_{0}\right)+\left\langle s_{k}\right\rangle\left(t_{0}\right)\right] \mathrm{e}^{-\left(t-t_{0}\right)}\right. \\
& \left.+\frac{2 \operatorname{sh} B}{\sqrt{\operatorname{sh}^{2} B+\mathrm{e}^{-4 K}}} \frac{\lambda_{+}^{N}-\lambda_{-}^{N}}{\lambda_{+}^{N}+\lambda_{-}^{N}}\left[1-\mathrm{e}^{-\left(t-t_{0}\right)}\right]\right\} .
\end{aligned}
$$

This equation has a simplified form as a consequence of assuming the transition probability dependent on one spin only. When the equilibrium magnetisation is zero, then $\left\langle s_{j} s_{k}\right\rangle(t)$ depends only on $\left\langle s_{j}\right\rangle(t)$ and $\left\langle s_{k}\right\rangle(t)$ and does not depend on the two-spin correlations.

The dynamics proposed can be easily adapted to the systems without the translational invariance. In order to do this we have to find $\boldsymbol{R}=\boldsymbol{M}^{m} \boldsymbol{Z} \boldsymbol{M}^{N-m}$ where $\boldsymbol{Z}$ is the transition matrix with the exchange integral zero. The transition probability is obtained in a similar way as for the system without ends and we can write 


$$
W\left(s_{i}\right)=\frac{1}{2}-\frac{1}{2} s_{i} \frac{\langle+|\boldsymbol{R}|+\rangle-\langle-|\boldsymbol{R}|-\rangle}{\langle+|\boldsymbol{R}|+\rangle+\langle-|\boldsymbol{R}|-\rangle}=\frac{1}{2}-\frac{1}{2} s_{i} r
$$

where

$$
\begin{aligned}
& \langle+|\boldsymbol{R}|+\rangle-\langle-|\boldsymbol{R}|-\rangle=\frac{2}{\mathrm{e}^{4 K}\left(\operatorname{sh}^{2} B+\mathrm{e}^{-4 K}\right)}\left[\lambda _ { + } ^ { N } \left(\mathrm{e}^{4 K} \operatorname{sh}^{3} B+\mathrm{e}^{2 K} \mathrm{sh} B\right.\right. \\
& \left.+\mathrm{e}^{4 K} \operatorname{sh} B \operatorname{ch} B \sqrt{\operatorname{sh}^{2} B+\mathrm{e}^{-4 K}}\right)+\lambda_{+}^{m} \lambda_{+}^{N-m} \operatorname{sh} B\left(1-\mathrm{e}^{2 K}\right) \\
& +\lambda_{-}^{m} \lambda_{+}^{N-m} \operatorname{sh} B\left(1-\mathrm{e}^{2 K}\right) \\
& \left.+\lambda_{-}^{N}\left(\mathrm{e}^{4 K} \operatorname{sh}^{3} B+\mathrm{e}^{2 K} \mathrm{sh} B-\mathrm{e}^{4 K} \operatorname{sh} B \operatorname{ch} B \sqrt{\operatorname{sh}^{2} B+\mathrm{e}^{-4 K}}\right)\right] \\
& \langle+|\boldsymbol{R}|+\rangle+\langle-|\boldsymbol{R}|-\rangle=\frac{2}{\mathrm{e}^{2 K}\left(\operatorname{sh}^{2} B+\mathrm{e}^{-4 K}\right)} \\
& \quad\left[\lambda_{+}^{N}\left(\mathrm{e}^{2 K} \mathrm{sh}^{2} B+1+\mathrm{e}^{2 K} \operatorname{ch}^{2} \sqrt{\operatorname{sh}^{2} B+\mathrm{e}^{-4 K}}\right)\right. \\
& \left.+\lambda_{-}^{N}\left(\mathrm{e}^{2 K} \operatorname{ch} B \sqrt{\operatorname{sh}^{2} B+\mathrm{e}^{-4 K}}-\mathrm{e}^{2 K} \operatorname{sh}^{2} B-1\right)\right]
\end{aligned}
$$

This transition probability permits formulation of the equation for the $i$-th site magnetisation changes, whose solution is similar to that obtained for the chain without ends

$$
\left\langle s_{i}\right\rangle(t)=\left\langle s_{i}\right\rangle\left(t_{0}\right) \mathrm{e}^{-\left(t-t_{0}\right)}+f(B, K, N, i, t) .
$$

In this equation the second term is related to the inhomogeneity of the differential equation and besides the earlier mentioned parameters $B, K, N$, $t$, it also depends on the distance of the $i$-th site from the edges.

In order to determine the relaxation of the mean spin, the system is divided into clusters. A cluster is made of $m$ nonzero exchange integrals and has zero exchange integrals at both ends. The probability of finding a cluster of $m$ bonds is $(1-p)^{2} p^{m}$, where $p$ is the concentration of nonzero exchange integrals. Denoting the total magnetisation of a cluster by $r_{m}(t)$, the mean magnetisation per spin can be found from the equation

$$
\left\langle\left\langle s_{i}\right\rangle\right\rangle(t)=(1-p)^{2} \sum_{m=0}^{\infty} r_{m}(t) p^{m} .
$$

As the influence of disorder is contained only in the inhomogeneous component (21), the relaxation time does not depend on the concentration $p$, which is a consequence of the fact that in the detailed balance condition (3) the summation was made over all spins except the $j$-th one. The dynamics will be improved if the two neighbouring spins are left out of the sum. It is the lowest change in the probabilities of transition which endows the probabilities with a dependence on $N$. When three sites are left out of the summation, the dependence on $N$ will be removed and the problems with BBGKY hierarchy will appear again. 


\section{Dynamics with a two-spin window}

Let us write the master equation for the case when we describe the behaviour of the $j$-th spin and the states of the two neighbouring spins have been left out of the summation

$$
\begin{aligned}
& \frac{\mathrm{d}}{\mathrm{d} t} P\left(s_{j}, t\right)=-\sum_{s_{j-1}= \pm 1} W\left(s_{j-1}, s_{j}\right) P\left(s_{j-1}, s_{j}, t\right) \\
& \quad+\sum_{s_{j-1}= \pm 1} W\left(s_{j-1},-s_{j}\right) P\left(s_{j-1},-s_{j}, t\right) \\
& \quad-\sum_{s_{j+1}= \pm 1} W\left(s_{j}, s_{j+1}\right) P\left(s_{j}, s_{j+1}, t\right) \\
& \quad+\sum_{s_{j+1}= \pm 1} W\left(s_{j},-s_{j+1}\right) P\left(-s_{j},-s_{j+1}, t\right) .
\end{aligned}
$$

As earlier we assume that the transition probability $W$ satisfies the detailed balance condition, so that

$$
\frac{W\left(s_{j-1}, s_{j}\right)}{W\left(s_{j-1},-s_{j}\right)}=\frac{P\left(s_{j-1},-s_{j}\right)}{P\left(s_{j-1}, s_{j}\right)} .
$$

Denoting by $\boldsymbol{O}$ the $4 \times 4$ transition matrix for two bonds, we can find its $n$-th power, used in the expressions for the probabilities of states of the two neighbouring spins

$$
P\left(s_{1}, s_{2}\right)=\frac{\left\langle s_{1}, s_{2}\left|\boldsymbol{O}^{N}\right| s_{1}, s_{2}\right\rangle}{\sum_{s_{1}= \pm 1} \sum_{s_{2}= \pm 1}\left\langle s_{1}, s_{2}\left|\boldsymbol{O}^{N}\right| s_{1}, s_{2}\right\rangle} .
$$

Equations (24) and (25) permit rewriting the equation for the transition probability in the form

$$
W\left(s_{j-1},-s_{j}\right)=\frac{1}{4}-s_{j-1} f-s_{j} g+s_{j-1} s_{j} h
$$

where

$$
\begin{aligned}
& f=g=-\frac{1}{4} \frac{\mathrm{e}^{K} \operatorname{sh} B}{\sqrt{\mathrm{e}^{2 K} \operatorname{sh}^{2} B+\mathrm{e}^{-2 K}}} \frac{\lambda_{+}^{2 N}-\lambda_{-}^{2 N}}{\lambda_{+}^{2 N}+\lambda_{-}^{2 N}}, \\
& h=\frac{1}{4 \operatorname{sh} 2 K}\left(\operatorname{ch} 2 K-\frac{\operatorname{ch} B}{\mathrm{e}^{K} \sqrt{\mathrm{e}^{2 K} \operatorname{sh}^{2} B+\mathrm{e}^{-2 K}}} \frac{\lambda_{+}^{2 N}-\lambda_{-}^{2 N}}{\lambda_{+}^{2 N}+\lambda_{-}^{2 N}}\right) .
\end{aligned}
$$

Multiplying (23) by $s_{j}$ and summing over the spin states we get

$$
\begin{aligned}
\frac{\mathrm{d}}{\mathrm{d} t}\left\langle s_{j}\right\rangle & =\frac{\mathrm{d}}{\mathrm{d} t} \sum_{s_{j}= \pm 1} s_{j} P\left(s_{j}, t\right) \\
= & -\sum_{s_{j-1}= \pm 1} \sum_{s_{j}= \pm 1} s_{j}\left(\frac{1}{4}-s_{j-1} f+s_{j} f-s_{j-1} s_{j} h\right) P\left(s_{j-1}, s_{j}, t\right) \\
& +\sum_{s_{j-1}= \pm 1} \sum_{s_{j}= \pm 1} s_{j}\left(\frac{1}{4}-s_{j-1} f-s_{j} f+s_{j-1} s_{j} h\right) P\left(s_{j-1},-s_{j}, t\right)
\end{aligned}
$$




$$
\begin{aligned}
& -\sum_{s_{j+1}= \pm 1} \sum_{s_{j}= \pm 1} s_{j}\left(\frac{1}{4}+s_{j} f-s_{j+1} f-s_{j} s_{j+1} h\right) P\left(s_{j}, s_{j+1}, t\right) \\
& +\sum_{s_{j+1}= \pm 1} \sum_{s_{j}= \pm 1} s_{j}\left(\frac{1}{4}-s_{j} f-s_{j+1} f+s_{j} s_{j+1} h\right) P\left(-s_{j}, s_{j+1}, t\right) \\
& =-\left\langle s_{j}\right\rangle-4 f+2 f\left(\left\langle s_{j-1} s_{j}\right\rangle+\left\langle s_{j} s_{j+1}\right\rangle\right)+2 h\left(\left\langle s_{j-1}\right\rangle+\left\langle s_{j+1}\right\rangle\right) .
\end{aligned}
$$

As follows, the equation for average spin besides the magnetisation terms contains also the spin correlations between the nearest neighbours and the problem can be solved only by solving a set of $2^{N}$ equations for each correlation function. However, we have not used hitherto the fact that the transition probability can be multiplied by an arbitrary function not containing the $j$-th spin and it would still lead the system to the equilibrium state, which follows from defining the transition probability through the detailed balance condition. Therefore,

$$
\begin{aligned}
& \tilde{W}\left(s_{j-1},-s_{j}\right)=W\left(s_{j-1},-s_{j}\right) F\left(s_{j-1}\right) \\
& \quad=\left(\frac{1}{4}-s_{j-1} f-s_{j} f+s_{j-1} s_{j} h\right)\left(1+s_{j-1} a\right) \\
& \quad=\left[\left(\frac{1}{4}-a f\right)-s_{j-1}\left(f-\frac{1}{4} a\right)-s_{j}(f-h a)+s_{j-1} s_{j}(h-f a)\right] \\
& \quad=b-s_{j} c+s_{j-1} s_{j} d .
\end{aligned}
$$

The latter equation was obtained by imposing the condition of the coefficient at $s_{j-1}$ being zero, then

$$
\begin{aligned}
b= & \frac{1}{4}-4 f^{2}=\frac{1}{4}\left[1-\frac{\mathrm{e}^{2 k} \operatorname{sh}^{2} B}{\mathrm{e}^{2 K} \operatorname{sh}^{2} B+\mathrm{e}^{-2 K}}\left(\frac{\lambda_{+}^{2 N}-\lambda_{-}^{2 N}}{\lambda_{+}^{2 N}+\lambda_{-}^{2 N}}\right)^{2}\right], \\
c= & f(1-4 h)=\frac{1}{4} \frac{\mathrm{e}^{K} \mathrm{sh} B}{\sqrt{\mathrm{e}^{2 K} \operatorname{sh}^{2} B+\mathrm{e}^{-2 K}}} \frac{\lambda_{+}^{2 N}-\lambda_{-}^{2 N}}{\lambda_{+}^{2 N}+\lambda_{-}^{2 N}} \\
& \times\left[\tanh K-\frac{\operatorname{ch} B}{\operatorname{sh} 2 K \mathrm{e}^{K} \sqrt{\mathrm{e}^{2 K} \operatorname{sh}^{2} B+\mathrm{e}^{-2 K}}} \frac{\lambda_{+}^{2 N}-\lambda_{-}^{2 N}}{\lambda_{+}^{2 N}+\lambda_{-}^{2 N}}\right], \\
d= & h-4 f^{2}=\frac{1}{4 \operatorname{sh} 2 K}\left(\operatorname{ch} 2 K-\frac{\operatorname{ch} B}{\mathrm{e}^{4 K} \sqrt{\mathrm{e}^{2 K} \operatorname{sh}^{2} B+\mathrm{e}^{-2 K}}} \frac{\lambda_{+}^{2 N}-\lambda_{-}^{2 N}}{\lambda_{+}^{2 N}+\lambda_{-}^{2 N}}\right) \\
& -\frac{1}{4} \frac{\mathrm{e}^{2 K} \operatorname{sh}^{2} B}{\mathrm{e}^{2 K} \operatorname{sh}^{2} B+\mathrm{e}^{-2 K}}\left(\frac{\lambda_{+}^{2 N}-\lambda_{-}^{2 N}}{\lambda_{+}^{2 N}+\lambda_{-}^{2 N}}\right)^{2} .
\end{aligned}
$$

The transition probability defined in this way leads to the equation for magnetisation

$$
\frac{\mathrm{d}}{\mathrm{d} t}\left\langle s_{j}\right\rangle=-4 b\left\langle s_{j}\right\rangle-4 c+2 d\left(\left\langle s_{j-1}\right\rangle+\left\langle s_{j+1}\right\rangle\right) .
$$

By putting $B=0$ we have $b=1 / 4, c=0$ and $d=\left[\operatorname{ch} 2 K-\left(\lambda_{+}^{2 N}-\lambda_{-}^{2 N}\right) /\left(\lambda_{+}^{2 N}+\right.\right.$ $\left.\left.\lambda_{-}^{2 N}\right)\right] /(4 \operatorname{sh} 2 K)$. The obtained equation differs from Eq. (30) proposed by 
Glauber [1] by the coefficient at $\left(\left\langle s_{i-1}\right\rangle+\left\langle s_{i+1}\right\rangle\right)$, and becomes identical to this equation for $N=1, d=\tanh 2 K / 4$. For any $B$, when dividing Eq. (29) by $4 b$ we get

$$
\frac{\mathrm{d}}{\mathrm{d}(4 b t)}\left\langle s_{j}\right\rangle=-\left\langle s_{j}\right\rangle-\frac{c}{b}+\frac{d}{2 b}\left(\left\langle s_{j-1}\right\rangle+\left\langle s_{j+1}\right\rangle\right) .
$$

This equation has the same form as Eq. (87) from [1] or (3.8) from [10], therefore, for $N \rightarrow \infty$ we can write the solution as

$$
\left\langle s_{i}\right\rangle(t)=\mathrm{e}^{-4 b\left(t-t_{0}\right)} \sum_{l}\left\langle s_{l}\right\rangle\left(t_{0}\right) I_{(j-l)}\left[4 d\left(t-t_{0}\right)\right]-\int_{t_{0}}^{t} 4 c \mathrm{e}^{-4(b-d)\left(t-t^{\prime}\right)} \mathrm{d} t^{\prime},
$$

where $I$ is the Bessel function of an imaginary argument

$$
\exp \left(\frac{x}{2}\left(\mu+\mu^{-1}\right)\right)=\sum_{n=-\infty}^{\infty} \mu^{n} I_{n}(x)
$$

When $B=0$ for the translationally invariant system we can write: $\left\langle s_{j}\right\rangle(t)=$ $\left\langle s_{j}\right\rangle\left(t_{0}\right) \mathrm{e}^{-4(b-d)\left(t-t_{0}\right)}$, while for a ferromagnet in low temperatures we can assume that $\lambda_{+}=2 \operatorname{ch} K \simeq 2 \operatorname{sh} K=\lambda_{-}$and $d=\operatorname{ch} 2 K /(4 \operatorname{sh} 2 K)$. In such conditions the relaxation time $\tau=1 /(4 b-4 d)$ does not depend on the number of sites $N$ and when $T \rightarrow 0, \tau \rightarrow \infty$, then the critical slowing down occurs. For an antiferromagnetic $d \rightarrow-\frac{1}{4}$ when $T \rightarrow 0$ then $\tau \rightarrow \frac{1}{2}$ and no critical slowing down takes place.

\section{Summary}

Two types of dynamics called the dynamics with single-spin window and two-spin window were proposed. These dynamics were formulated on the basis of the definitions of the transition probability based on the detailed balance condition summed over the states. Although this artificial assumption weakens the dynamics of the system, it enables analytical investigation of the dynamics. In Sect. 3 only the ordered model was analysed, but the analysis can be adapted to the disordered case, according to the scheme given in Sect. 2. As the relaxation time for the dynamics with a two-spin window depends on the number of spins in the chain, for a disordered system it will depend on the concentration.

\section{References}

[1] R.J. Glauber, J. Math. Phys. 4, 294 (1963).

[2] D. Dhar, M. Barma, J. Stat. Phys. 22, 259 (1980).

[3] H. Falk, Physica A 117, 561 (1983).

[4] L.E. Reichl, A Modern Course in Statistical Physics, Edward Arnold Ltd, University of Texas Press, Austin 1980, p. 193.

[5] J.V. José M. Mehl, J. Sokoloff, Phys. Rev. B 27, 434 (1982).

[6] G. Forgacs, D. Mukamel, R.A. Pelcovits, Phys. Rev. B 30, 205 (1984).

[7] J.J. Luque, A. Cordoba, Physica A 131, 348 (1985).

[8] H.J. Hilhorst, Physica A 97, 171 (1975). 
[9] A. Baumgärtner, K. Binder, J. Stat. Phys. 18, 423 (1978).

[10] D. Das, M. Barma, Phys. Rev. E 60, 2577 (1999).

[11] M. Suzuki, R. Kubo, J. Phys. Soc. Japan 24, 51 (1968). 